\title{
Improved trailing edge noise prediction using a generalized Rapid-distortion theory approach
}

\author{
M. Z. Afsar ${ }^{1}$ \\ Department of Mechanical \& Aerospace Engineering, University of Strathclyde, Glasgow G1 1XJ
}

\begin{abstract}
Goldstein, Afsar \& Leib (J. Fluid Mech., vol. 736, pp. 532-569, 2013) and Goldstein-LeibAfsar (J Fluid Mech. vol. 824, pp. 477-512) extended the Rapid-distortion theory (RDT) of turbulence to consider mean flows that are transversely sheared in the upstream state. The key feature of the theory was in relating the upstream boundary condition to physically realizable turbulence that can be controlled by an experimentalist. We summarize the theory briefly illustrating how it can be used to model trailing edge noise. We conclude by correcting the high frequency formula that GLA derived to include the next order term for the amplitude function in the WKBJ approximation for the scattered pressure. This term enters the lowest order expansion for the scattered pressure when the hydrodynamic wave number limit is taken. The predictions based on this mathematical representation have greater flexibility at high frequencies.
\end{abstract}

\section{Introduction}

Rapid-distortion theory uses linear analysis to study the interaction of turbulence with solid surfaces. For example, jet-surface interaction noise in Fig. 1 occur when surfaces play a direct role in the generation of sound and/or its propagation.

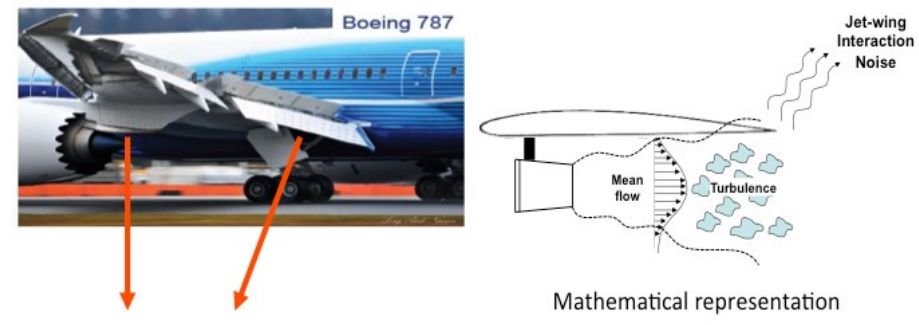

Fig. 1 Modeling edge noise

\section{Basic Scalings}

RDT applies when the turbulence intensity is taken as a global small parameter everywhere in the flow; i.e. $\alpha \square|\boldsymbol{u}| / U_{J} \square O(1)$ where $\alpha$ is the turbulence intensity and $|\boldsymbol{u}|$ is the magnitude of the local rms turbulence velocity and $U_{J}$ is some characteristic reference velocity. It also implies that the length (or time) scale over which the interaction takes place is short compared to the length (or time) scale over which the turbulent eddies evolve. Hence, when interpreted asymptotically, these assumptions imply, that it is possible to identify a distance that is very (infinitely) large on the scale of the interaction, but still small on the scale over which the turbulent eddies evolve. The assumptions imply that the resulting flow is inviscid and non-heat-conducting and is, therefore, governed by the linearized Euler equations linearized about an arbitrary base flow.

RDT can be thought of as a lowest order perturbation of the Navier-Stokes equations in the small turbulence intensity, $\alpha$, in the vicinity of the trailing edge. The problem is, therefore, linear and inviscid and the mean flow

\footnotetext{
${ }^{1}$ Chancellor's Fellow, and AIAA Member
} 
near the trailing edge is nearly transversely sheared. The inviscid pressure perturbation, $p^{\prime}=p-\bar{p} \&$ mass flux $\boldsymbol{u} \square \rho \boldsymbol{v}^{\prime}$, (where $\boldsymbol{v}^{\prime}$ denotes the velocity perturbation) are governed by the leading-order momentum and energy equations:

$$
\begin{gathered}
\frac{D_{0} u_{i}}{D \tau}+\delta_{1 i} u_{j} \frac{\square U}{\square y_{j}}+\frac{\square p^{\prime}}{\square y_{i}}=0 \\
\frac{D_{0} p^{\prime}}{D \tau}+\frac{\square c^{2} u_{j}}{\square y_{j}}=0
\end{gathered}
$$

that are linearized about the transversely mean flow, $U\left(\boldsymbol{y}_{T}\right)$, and mean sound speed squared $c^{2}\left(\boldsymbol{y}_{T}\right)$ and which reduces to the compressible Rayleigh equation $L p^{\prime}=0$. Using the direct Green's function of Rayleigh equation as an adjoint, GAL-13 used Green's theorem (Morse and Feshbach, 1953, p. 870, Tam and Auriault, 1998) to show the Euler equations possess basic solutions

$$
\begin{gathered}
p^{\prime}(\boldsymbol{x}, t)=\frac{D_{0}^{3}}{D t^{3}} \int_{-T}^{T} \int_{V(\boldsymbol{y})} g(\boldsymbol{y}, \tau \mid \boldsymbol{x}, t) \tilde{\omega}_{c}\left(\tau-y_{1} / U\left(\boldsymbol{y}_{T}\right), \boldsymbol{y}_{T}\right) d \boldsymbol{y} d \tau \\
\rho v_{\perp}^{\prime}=-\frac{\partial U / \partial x_{i}}{|\nabla U|} \int_{-T}^{T} \int_{V(\boldsymbol{y})} g_{i}(\boldsymbol{y}, \tau \mid \boldsymbol{x}, t) \tilde{\omega}_{c}\left(\tau-y_{1} / U\left(\boldsymbol{y}_{T}\right), \boldsymbol{y}_{T}\right) d \boldsymbol{y} d \tau
\end{gathered}
$$

respectively where $T$ denotes a very large, but finite, time interval. The solid surfaces $S(\boldsymbol{y})$ bound volume $V(\boldsymbol{y})$ in these formulas can be finite, semi-infinite or infinite in the streamwise direction but must coincide with any level surfaces of the mean velocity profile. $g_{i}(\boldsymbol{y}, \tau \mid \boldsymbol{x}, t)$ is linearly related to $g(\boldsymbol{y}, \tau \mid \boldsymbol{x}, t)$, which is determined for incoming wave behavior as $|\boldsymbol{y}| \rightarrow \infty$ and appropriate boundary conditions on $S(\boldsymbol{y})$.

\section{A. Local relations for $\tilde{\omega}_{c}$}

Since $\tilde{\omega}_{c}$ satisfies $D_{0} \tilde{\omega}_{c} / D \tau \equiv 0$ by definition, for an arbitrary selection of itsarguments, it can be used to specify the upstream boundary condition (input) within a boundary value problem (BVP) that seeks to determine acoustic spectrum as its "output". The upstream boundary condition must obviously be independent of the local scattered flow near the the trailing edge. Hence it must be imposed at large (infinite) distances (say $x_{b c} \rightarrow \infty$ ) away from it. Although in a strict asymptotic sense, $x_{b c}$, lies between the $\mathrm{O}(1)$ shear layer growth scale and the eddy length scale over which the interaction takes place. The latter can be taken to infinity since these lengths become disparate in the RDT limit.

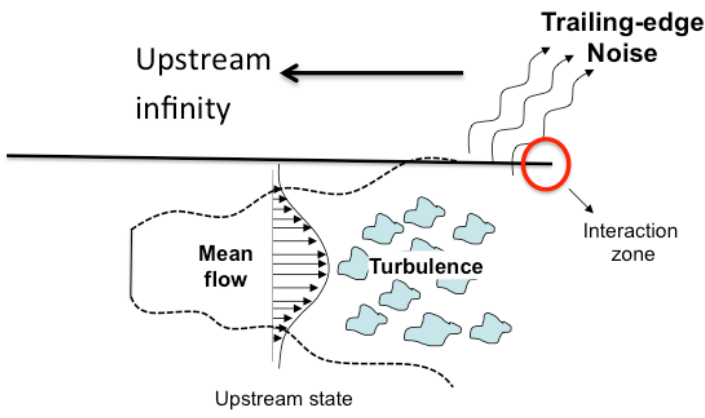

Fig. 2 Upstream boundary

\section{Condition}


That is, since the shear layer grows over infinitely long distances, $x$, when the interaction length scale, $\bar{x}$, of the eddy is fixed at $\mathrm{O}(1), x_{b c}$ lies within bound: $\bar{x} \square x_{b c} \square x$. But since the ratio of these length scales becomes asymptotically disparate in the RDT limit as: $\frac{\bar{x}}{x}=\frac{\tau_{\text {turnover }}}{\tau_{\text {int }}}:=\frac{1}{\varepsilon} \rightarrow \infty$ as $\varepsilon \rightarrow 0$ when the (non-dimensional) the shear layer growth scale, $x=U_{c} / \tau_{\text {turnover }}=O(1)$ the (non-dimensional) eddy length scale over which the interaction takes place can still be taken at infinity $\bar{x}=U_{c} / \tau_{\text {int }} \rightarrow \infty \square \varepsilon \bar{x}=O(1)$. Goldstein-Leib-Afsar (2017, JFM) showed that when $\tilde{\omega}_{c}$ is determined under this far upstream limit, the particle displacement term and pressure term in its general evolution equation (derived in Goldstein-Afsar-Leib 2013b) drops out (i.e. become asymptotically small).

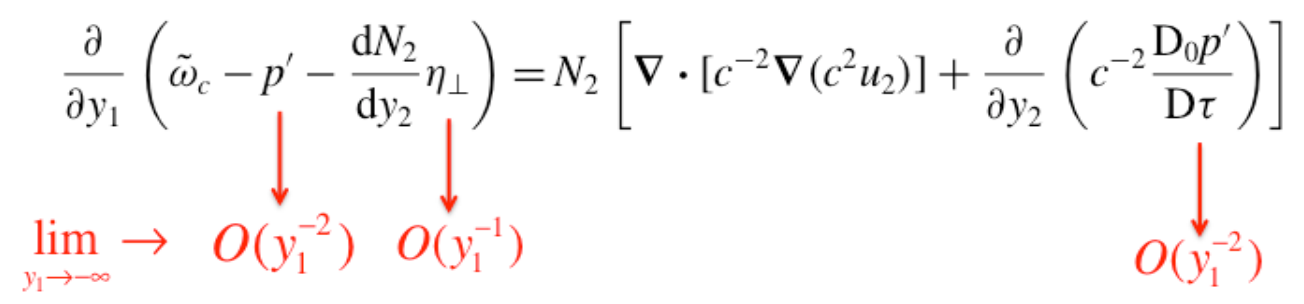

The far-field asymptotes above show, among other things, that there is a direct algebraic relation between $\tilde{\omega}_{c}$ and the physically measurable turbulence properties which can be expressed in terms of the transverse velocity whose correlation has been extensively measured and whose functional form is well known (Afsar-Leib-Bozak JSV 2017).

Allowing satisfies causality.

\section{B. Correspondence relation to far upstream hydrodynamics}

The correspondence relation derived in GLA17 shows that the Fourier transform:

$$
\hat{\boldsymbol{\Omega}}_{c}\left(y_{2} ; \omega, k_{3}, T\right)=\frac{1}{(2 \pi)^{2}} \int_{-T}^{T} e^{-i y_{3} k_{3}} \int_{-T}^{T} e^{i \omega \xi} \tilde{\omega}_{c}\left(\xi, \boldsymbol{y}_{T}\right) d \xi d y_{3}
$$

is related to the commensurate double Fourier transform

$$
\left.\hat{\Gamma}_{\infty}\left(y_{2} ; \omega, k_{3}, T\right)=\frac{1}{(2 \pi)^{2}} \lim _{y_{1} \rightarrow-\infty} \int_{-\infty}^{\infty} e^{-i y_{3} k_{3}} \int_{-T}^{T} e^{i \omega\left[\tau-y_{1} / U\left(\boldsymbol{y}_{T}\right)\right.}\right] \Gamma_{2,0}(\boldsymbol{y}, \tau) d \tau d y_{3}
$$

of the vorticity derivative $\lim _{y_{1} \rightarrow-\infty} \Gamma_{2,0}=\nabla \cdot\left[c^{-2} \nabla\left(c^{2} u_{2}\right)\right]$ by the remarkably simple connectivity formula: 
$\hat{\Omega}_{c}\left(y_{2}: \omega, k_{3}, T\right)=\frac{1}{\mathrm{i} \omega} U\left(y_{2}\right) N_{2} \hat{\Gamma}_{\infty}$,

This provides a rationally based relation between the acoustic field and a near field measurement that can actually be made.

C. Physical Realizability We model the two point space-time correlation of the vorticity derivative $\Gamma_{2,0}(\boldsymbol{y}, \tau)$ by a functional form of the following type (Afsar+Leib+Bozak JSV 2017) shown below in Fig. 3.

$A\left(y_{2}, \tilde{y}_{2}\right) \rho\left(y_{2}\right) \rho\left(\tilde{y}_{2}\right)\left\{1+a_{1}\left(\tilde{\tau}-\left[\tilde{y}_{1} / U\left(\tilde{y}_{2}\right)-y_{1} / U\left(y_{2}\right)\right]\right) \frac{\partial}{\partial \tilde{\tau}}\right\} \longrightarrow \longrightarrow$ Responsible for $\times \exp -\sqrt{\left[f\left(\eta_{2} / l_{2}\right)\right]^{2}+\left\{\tilde{\tau}-\left[\tilde{y}_{1} / U\left(\tilde{y}_{2}\right)-y_{1} / U\left(y_{2}\right)\right]\right\}^{2} / \tau_{0}^{2}+\left(\eta_{3} / l_{3}\right)^{2}}$,

Responsible for cusp

in the auto-correlation

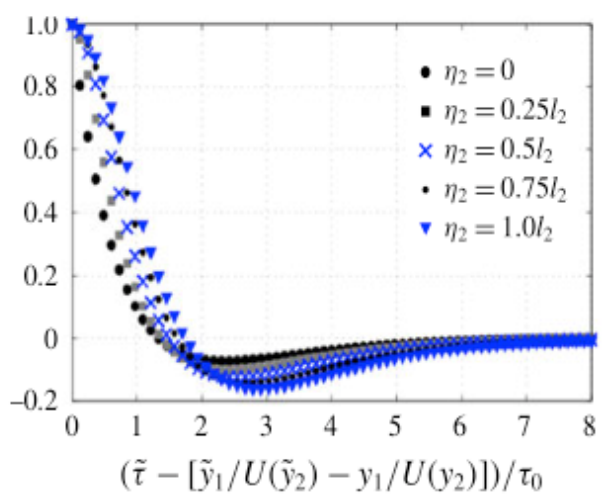

Fig. 3 Anti-correlations

For the rectangular jet that GLA and GAL considered, the auto-covariance $S\left(y_{2}, \tilde{y}_{2} ; k_{3}, \omega\right)$ of the Fourier transform $\hat{\Omega}_{c}\left(y_{2} ; \omega, k_{3} ; T\right)$ given formally by

$$
\begin{aligned}
& S\left(y_{2}, \tilde{y}_{2} ; k_{3}, \omega\right) \equiv(2 \pi)^{3} \lim _{T \rightarrow \infty} \frac{\hat{\Omega}_{c}\left(y_{2} ; \omega, k_{3} ; T\right) \hat{\Omega}_{c}^{*}\left(\tilde{y}_{2} ; \omega, k_{3} ; T\right)}{2 T} \\
& =\frac{1}{2 \pi} \int_{-\infty}^{\infty} \int_{-\infty}^{\infty} e^{i\left(\omega \tilde{\tau}-k_{3} \eta_{3}\right)}\left\langle\tilde{\omega}_{c}\left(t, \boldsymbol{y}_{T}\right) \tilde{\omega}_{c}\left(t+\tilde{\tau}, \tilde{y}_{2}, y_{3}+\eta_{3}\right)\right\rangle d \tilde{\tau} d \eta_{3}=\frac{U\left(y_{2}\right) U\left(\tilde{y}_{2}\right) N_{2} \tilde{N}_{2}}{2 \pi \omega^{2}} \\
& \quad \times \int_{-\infty}^{\infty} \int_{-\infty}^{\infty} e^{\left\{i\left[\omega\left(\tilde{\tau}-\left[\tilde{y}_{1} / U\left(\tilde{y}_{2}\right)-y_{1} / U\left(y_{2}\right)\right]\right)-k_{3} \eta_{3}\right]\right\}}\left\langle\Gamma_{\infty}(\boldsymbol{y}, t) \Gamma_{\infty}\left(\tilde{y}_{1}, \tilde{y}_{2}, y_{3}+\eta_{3}, t+\tilde{\tau}\right)\right\rangle d \tilde{\tau} d \eta_{3}
\end{aligned}
$$


for the two-dimensional planar mean flow $U\left(y_{2}\right)$ where $N_{2}$ is the scaled unit-vector defined via $N_{i} \equiv\left(\widetilde{c^{2}} /|\nabla U|^{2}\right)\left(\partial U / \partial y_{i}\right), \widetilde{c^{2}}$ is the Favre-averaged mean square speed of sound and $\hat{\Omega}_{c}\left(y_{2}: \omega, k_{3}, T\right)$ is defined above.

GLA showed that $S\left(y_{2}, \tilde{y}_{2} ; k_{3}, \omega\right)$ can be easily determined when the model for the vorticity derivative $\Gamma_{2,0}(\boldsymbol{y}, \tau)$ of the type shown in Fig. 3 is inserted into the Fourier transform:

$$
\bar{\Gamma}_{2,0}^{\infty}(\boldsymbol{y}: \omega, T)=\bar{\Gamma}_{2,0}^{\infty}\left(\boldsymbol{y}_{T}: \omega, T\right)=\frac{1}{2 \pi} \int_{-T}^{T} e^{i \omega\left[\tau-y_{1} / U\left(\boldsymbol{y}_{T}\right)\right]} \Gamma_{\infty}(\boldsymbol{y}, \tau) d \tau
$$

The final result they arrive at is:

$$
\begin{aligned}
S\left(y_{2}, \tilde{y}_{2} ; k_{3}, \omega\right)=-\left(\rho_{\infty} c_{\infty}^{2}\right)^{2} \tau_{0} l_{2}^{4} l_{3} A\left(y_{2}, \tilde{y}_{2}\right)\left[\frac{d U}{d y_{2}} \frac{d U}{d \tilde{y}_{2}} \frac{\omega^{2}}{U^{3}\left(\tilde{y}_{2}\right) U^{3}\left(y_{2}\right)}\right] \\
\times \\
\times\left[1-a_{1}\left(1+\frac{\left(\omega \tau_{0}\right)^{2}}{\chi} \frac{\partial}{\partial \chi}\right)+\ldots\right] \frac{1}{\chi} \frac{\partial}{\partial \chi}\left(\frac{\mathrm{e}^{-\left|f\left(\eta_{2} / l_{2}\right)\right| \chi}}{\chi}\right)
\end{aligned}
$$

where $\omega$ is the temporal frequency,

$$
\chi \equiv \sqrt{1+\left(\omega \tau_{0}\right)^{2}+\left(k_{3} l_{3}\right)^{2}}
$$

and where $f\left(\eta_{2} / 1_{2}\right)$ is a tranverse decay function.

\section{GLA17 predictions and extension to CFD data}

Splitting the Green's function into a particular (hydrodynamic part) defined on all space (doubly infinite streamwise plane) and a homogeneous scattered part that satisfies appropriate conditions on a half-plane \& can be determined by the Wiener-Hopf technique allows the far field acoustic spectrum to be determined (see GAL13). GAL analyzed the model problem shown in figure 5 of a jet emanating from a large-aspect ratio rectangular nozzle interacting with the trailing edge of a flat plate. The sound generated by this interaction was compared to recent experiments on this configuration that were performed at NASA Glenn Research Center (Zaman, Brown and Bridges 2013; Brown, 2015). The predictions shown in Fig. 4 taken from GLA paper show remain within reasonable agreement with data. 

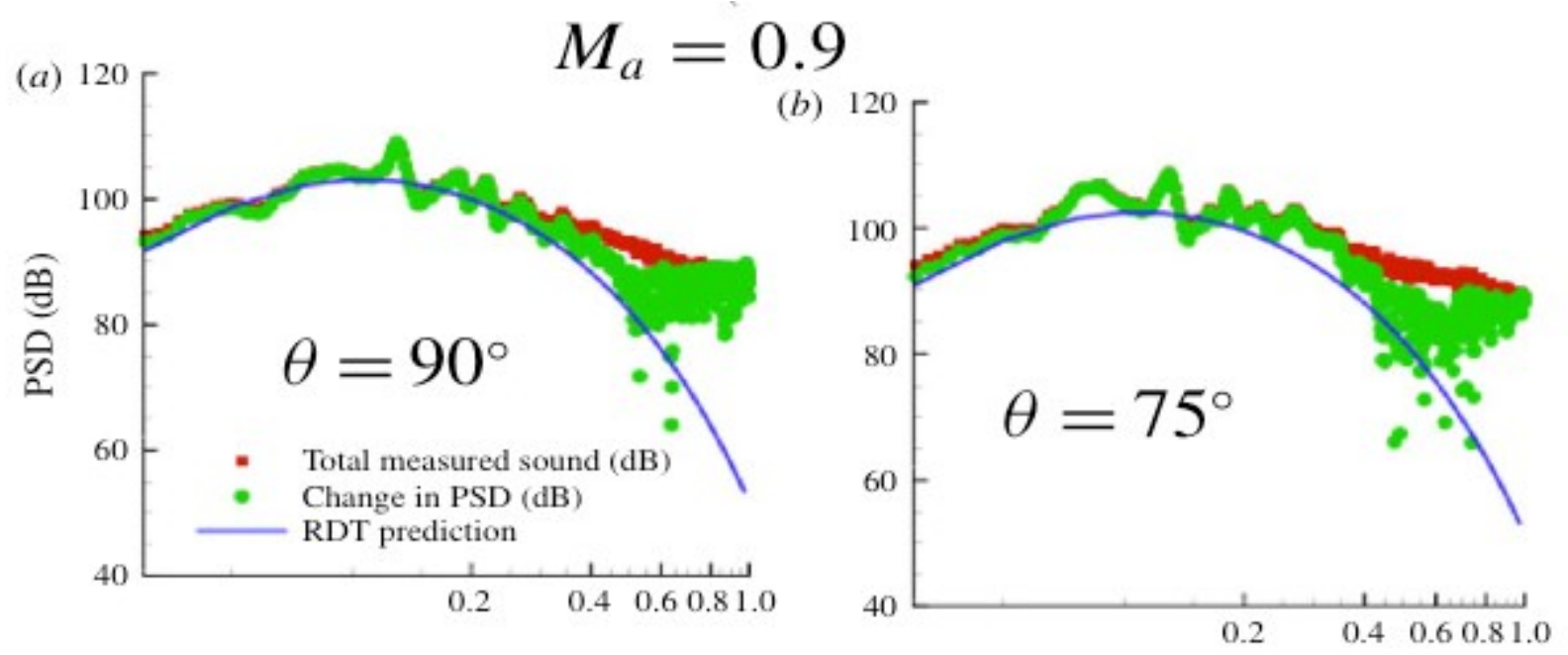

Fig. 4 GLA17 predictions. Power Spectral Density (PSD) of the far-field pressure fluctuations at 100 equivalent diameters from nozzle exit (lossless in $\mathrm{dB}$ scale referenced to $20 \mu \mathrm{Pa}$ ) as a function of Strouhal number, for $M_{a}=0.9$. Predicted (solid line): Measured data below the plate at $\psi=-90^{\circ}$. (Total noise: Red; difference between the total noise and noise measured in the free jet: Green.) Plate trailing edge at $\left(x_{d}, y_{d}\right) / D=(5.7,0.98)$ (a). $\theta=90^{\circ}$; (b) $\theta=75^{\circ}$ (c) $\theta=60^{\circ}$ (d) $\theta=45^{\circ}$

(See caption of Fig. 8 in GLA).
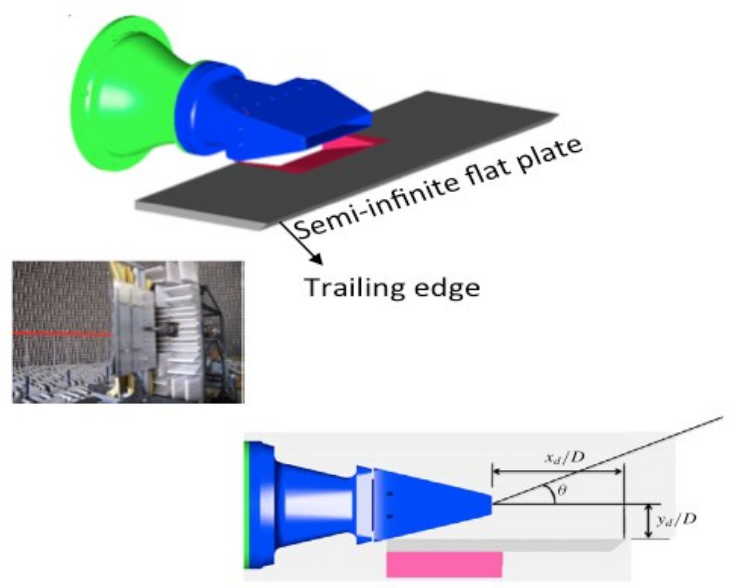

Fig. 5 NASA GRC jet-surface interaction Experiment (Zaman et al. 2013; Brown 2015)

\section{Correction to the GLA result}

GLA showed that the acoustic spectrum for the scattered pressure is given by

$$
I_{\omega}(\boldsymbol{x}) \equiv \frac{1}{2 \pi} \int_{-\infty}^{\infty} e^{i \omega \tilde{\tau}} \overline{p^{s}(\boldsymbol{x}, t) p^{s}(\boldsymbol{x}, t+\tilde{\tau})} d \tilde{\tau}
$$

where $\tilde{\bullet}$ denotes the time average, is given by

$$
I_{\omega}(\boldsymbol{x})=\left(\frac{(2 \pi)^{2} k_{\infty} \sin \theta \sin \psi}{|\boldsymbol{x}|}\right)^{2} \int_{0}^{\infty} \int_{0}^{\infty} \Phi_{\lessgtr}\left(k_{1}^{(s)}, k_{3}^{(s)}, y_{2}, \omega\right) \Phi_{\lessgtr}^{*}\left(k_{1}^{(s)}, k_{3}^{(s)}, \tilde{y}_{2}, \omega\right)
$$




$$
\times \bar{G}\left(y_{2} \mid 0 ; k_{3}^{(s)}, \omega / U\left(y_{2}\right), \omega\right) \bar{G}^{*}\left(\tilde{y}_{2} \mid 0 ; k_{3}^{(s)}, \omega / U\left(\tilde{y}_{2}\right), \omega\right) S\left(y_{2}, \tilde{y}_{2} ; k_{3}^{(s)}, \omega\right) d y_{2} d \tilde{y}_{2},
$$

for $x_{2} \lessgtr 0$ where $S\left(y_{2}, \tilde{y}_{2} ; k_{3}^{(s)}, \omega\right)$ is defined by (9).

The stationary points in wavenumber are given by $k_{1}^{(s)}=k_{\infty} \cos \theta, \quad k_{3}^{(s)}=k_{\infty} \sin \theta \cos \psi \quad$ where $k_{\infty} \equiv \omega / c_{\infty}$ is far-field wave number. The spectral function

$$
\Phi_{\lessgtr}\left(k_{1}, k_{1}, y_{2}, \omega\right) \equiv \frac{\kappa_{-}\left(k_{1}, k_{3}, \omega\right) A_{\lessgtr}}{\kappa_{+}\left(\omega / U\left(y_{2}\right), k_{3}, \omega\right)\left[\omega / U\left(y_{2}\right)-k_{1}\right] \hat{P}_{\lessgtr}^{\prime}\left(0 ; k_{1}, k_{3}, \omega\right)}
$$

is defined by

$$
\frac{A_{<}}{\hat{P}_{<}^{\prime}\left(0 ; k_{1}, k_{3}, \omega\right)}=\frac{1}{\sqrt{k_{1}^{2}+k_{3}^{2}-k_{\infty}^{2}}}
$$

where

$$
\bar{G}\left(y_{2} \mid 0 ; k_{3}^{(s)}, \omega / U\left(y_{2}\right), \omega\right)=\frac{\omega^{2} P_{>}\left(y_{2} ; \omega, \omega / U\left(y_{2}\right), k_{3}^{(s)}\right)}{(2 \pi)^{3} c_{s}^{2} P_{>}^{\prime}\left(0 ; \omega, \omega / U\left(y_{2}\right), k_{3}^{(s)}\right)} .
$$

$\kappa_{ \pm}\left(k_{1}, k_{3}, \omega\right)$ denote bounded analytic functions in the upper/lower half planes that satisfy the factorization condition

$$
\frac{\kappa_{+}\left(k_{1}, k_{3}, \omega\right)}{\kappa_{-}\left(k_{1}, k_{3}, \omega\right)}=\frac{P_{>}\left(0: \omega, k_{1}, k_{3}\right)}{P_{>}^{\prime}\left(0: \omega, k_{1}, k_{3}\right)}-\frac{1}{\sqrt{k_{1}^{2}+k_{3}^{2}-k_{\infty}^{2}}}
$$

$P_{\lessgtr}\left(y_{2} ; \omega, k_{1}, k_{3}\right)$ denote homogeneous solutions to two dimensional Rayleigh equation that has outgoing wave behavior as $y_{2} \rightarrow \square \infty, \theta$ denotes the polar angle measured from the downstream $x_{1}$ axis and $\psi$ denotes the azimuthal angle measured from the plane of the plate. GLA showed that the high frequency spectrum can be described by using the WKBJ method to obtain the high frequency outgoing wave homogeneous solution

$$
P_{>}\left(y_{2}: \omega, k_{1}, k_{3}\right)=\frac{1-\hat{k}_{1} M\left(y_{2}\right)}{\left[q\left(y_{2} \mid \hat{k}_{1}, \hat{k}_{3}\right)\right]^{1 / 4}} \exp \left(i k_{\infty} \int_{0}^{y_{2}} \sqrt{q\left(y \mid \hat{k}_{1}, \hat{k}_{3}\right)} d y\right)
$$

and therefore acoustic spectrum formula:

$$
\begin{array}{r}
I_{\omega}(\boldsymbol{x})=\left(\frac{k_{\infty}}{4 \pi|\boldsymbol{x}|}\right)^{2}(\beta-\cos \theta) \int_{0}^{\infty} \int_{0}^{\infty} \frac{\left[M\left(y_{2}\right) M\left(\tilde{y}_{2}\right)\right]^{3 / 2} Q\left(y_{2} \mid \theta, \varphi\right)\left[Q\left(\tilde{y}_{2} \mid \theta, \varphi\right)\right]^{*}}{\left[1-M\left(y_{2}\right) \cos \theta\right]\left[1-M\left(\tilde{y}_{2}\right) \cos \theta\right]} \\
\times \frac{S\left(y_{2}, \tilde{y}_{2} ; k_{3}^{(s)}, \omega\right)}{\sqrt{\left[1-\beta M\left(y_{2}\right)\right]\left[1-\beta M\left(\tilde{y}_{2}\right)\right]}} d y_{2} d \tilde{y}_{2},
\end{array}
$$

for $x_{2}<0$ where 


$$
Q\left(y_{2} \mid \theta, \varphi\right) \equiv\left[\frac{q\left(0 \mid y_{2}\right)-e^{-\chi_{0} k_{\infty}}}{q\left(y_{2} \mid y_{2}\right)}\right]^{1 / 4} \exp \left(i k_{\infty} \int_{0}^{y_{2}} \sqrt{q\left(y \mid y_{2}\right)} d y\right)
$$

$\beta \square\left(1-\sin ^{2} \theta \cos ^{2} \psi\right)^{1 / 2}, M\left(y_{2}\right)=U\left(y_{2}\right) / c_{\square}$ denotes the local acoustic Mach number at the position $y_{2}$ and $\chi_{0}$ is a positive constant and we have inserted the exponential damping factor $e^{-\chi_{0} k_{\infty}}$ into (19), which leaves the asymptotic expansion unchanged to the order of approximation considered here. $S\left(y_{2}, \tilde{y}_{2} ; k_{3}^{(s)}, \omega\right)$ is given by (9).

However, the next order term for amplitude expansion in the WKBJ approximation (17) enters the lowest order solution when the hydrodynamic wavenumber limit is taken inasmuch as $\Phi\left(\hat{k} ; y_{2}\right)=1-\hat{k}_{1} M\left(y_{2}\right) \rightarrow 0$ when $\hat{k} \rightarrow 1 / M\left(y_{2}\right)$.

The result that we obtain (discused in the presentation at the conference) is then given by (18) but where now:

$$
Q\left(y_{2} \mid \theta, \varphi\right) \equiv \frac{1}{1+i k_{\infty} \chi_{1} a_{r}\left(y_{2}\right)\left(\frac{M\left(y_{2}\right)}{M^{\prime}\left(y_{2}\right)}\right) q^{1 / 2}\left(y_{2} \mid y_{2}\right)}\left[\frac{q\left(0 \mid y_{2}\right)-e^{-\chi_{0} k_{\infty}}}{q\left(y_{2} \mid y_{2}\right)}\right]^{1 / 4} \exp \left(i k_{\infty} \int_{0}^{y_{2}} \sqrt{q\left(y \mid y_{2}\right)} d y\right)
$$

where $\chi_{1}$ is another constant that continues to render (20) asymptotically equivalent to the straight forward low frequency result given by (6.33) of GAL in which $Q\left(y_{2} \mid \theta, \varphi\right)=1$ because $Q\left(y_{2} \mid \theta, \varphi\right)$ also $\rightarrow 1$ in (20) as $k_{\infty} \rightarrow 0$. The additional factors in (20) are defined as:

$$
q\left(y \mid \hat{k}_{1}, \hat{k}_{3}\right) \equiv\left[1-\hat{k}_{1} M(y)\right]^{2}-\hat{k}_{1}^{2}-\hat{k}_{3}^{2}
$$

where

$$
q\left(y \mid y_{2}\right) \square q\left(y \mid 1 / M\left(y_{2}\right), \sin \theta \cos \psi\right)
$$

and $a_{r}\left(y_{2}\right)=\widetilde{c^{2}}\left(y_{2}\right) / c_{\infty}^{2}$ is the Favre-averaged mean square speed of sound ratio.

Predictions based on (20) (after inserting this into (18) and using (9) for $S\left(y_{2}, \tilde{y}_{2} ; k_{3}^{(s)}, \omega\right)$ give greater flexibilty at high frequencies since the $\chi_{1}=O(1)$ parameter governs the rate at high frequency decay of the acoustic spectrum. In Fig. 6 we show the compare the $90^{\circ}$ prediction obtained by GLA to that via (18) \& (19) for SP07 (Ma= 0.9 ) jet at the same trailing edge location as in Fig. 4 (see caption of Fig. 8 in GLA). Further comparisons will be made for the presentation at the conference. 


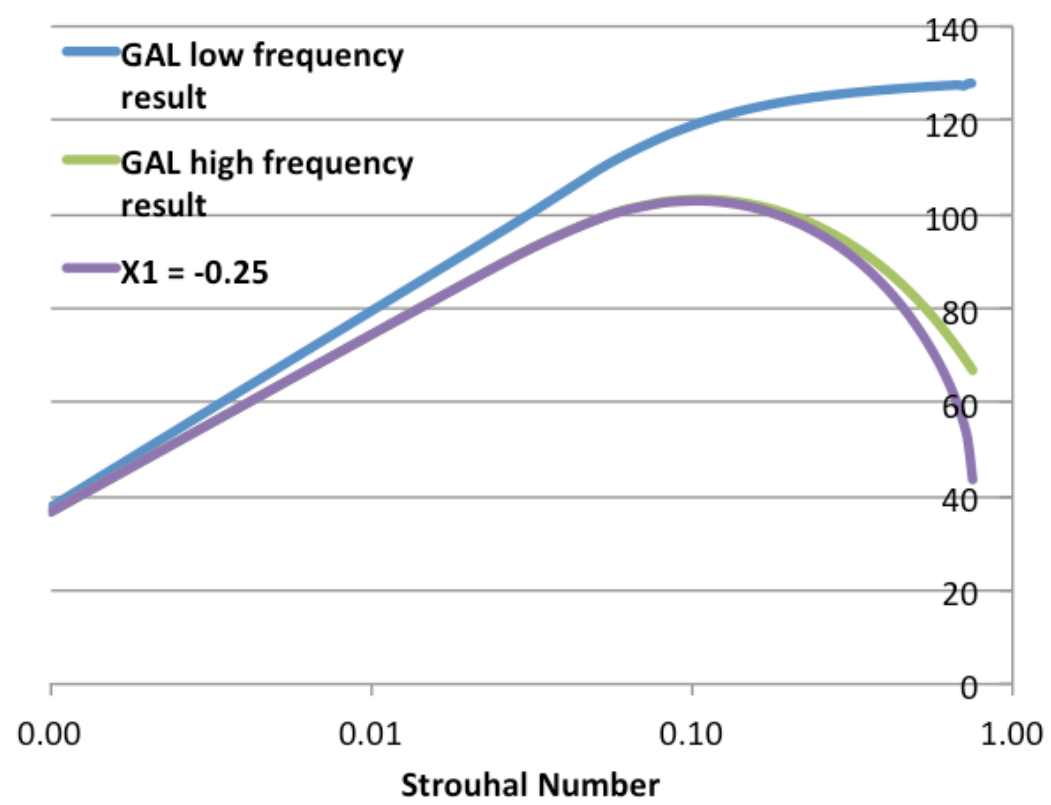

Fig. 6 Comparison of GLA17 predictions to that obtained using (18) \& (19). Caption as in Fig. 4 (or Fig. 8 in GLA). Purple curve shows faster high frequency decay possible using (18) \& (20).

\section{Conclusions}

The GAL/GLA rapid-distortion theory shows that the upstream turbulence does not convect with the flow in the RDT-limit when the upstream base flow is non-uniform (transversely sheared); i.e.

$$
\frac{\mathrm{D}_{0}}{\mathrm{D} \mathbb{x}} \bar{c}(x: !) \square 0
$$

But the convected quantity $\tilde{\omega}_{c}$ is: $D_{0} \tilde{\omega}_{c} / D \tau \equiv 0$ And it is related to the upstream physics in the manner described. The high frequency correction given by (20) will be further discussed during the conference presentation with more comparisions made to the GLA results.

\section{References}

[1] Goldstein, M.E., Afsar, M. Z. and Leib, 2013 a, Non-homogeneous rapid distortion theory on transversely sheared mean flows, J. Fluid Mech. Vol.736, pp. 532-569

[2] Goldstein, M.E., Leib, S. J. and Afsar, M. Z. 2017 a, Generalized rapid distortion theory on transversely sheared mean flows with physically realizable upstream boundary conditions: application to the trailing edge problem, J. Fluid Mech. Vol.824, pp. 477-512

[3] Goldstein, M.E., Afsar, M. Z. and Leib, 2013 b, Structure of Small Amplitude Motion on Transversely Sheared Mean Flows, NASA/TM-2013-217862

[4]. Afsar, M.Z., Leib, S.J. and Bozak, R.F., 'Effect of de-correlating turbulence on the low frequency decay of jet-surface interaction noise in sub-sonic unheated air jets using a CFD-based approach,' Journal of Sound and Vibration, Vol. 386, No. 6, pp. 177-207, 2017. 
[5]. Brown, Clifford A., 2015, An Empirical Jet-Surface Interaction Noise Model with Temperature and Nozzle Aspect Ratio Effects, 53rd AIAA Aerospace Sciences Meeting.

[6]. Zaman, K.; Brown, C. A.; Bridges, J. E., 2013, Interaction of a Rectangular Jet with a Flat-plate placed parallel to the Flow AIAA-2013-2184. (NASA/TM-2013-217879 (E-18684), 2013. 